\title{
Development of tartaric esters as bifunctional additives of methanol-gasoline
}

\author{
Jie Zhang ${ }^{1}$, Changchun Yang ${ }^{1,2}$, Ying Tang ${ }^{1 *}$, Rui Zhou ${ }^{1}$, Xiaoli Wang ${ }^{3}$ and Lianghong $\mathrm{Xu}^{3}$
}

\begin{abstract}
Background: Methanol has become an alternative fuel for gasoline, which is facing a rapidly rising world demand with a limited oil supply. Methanol-gasoline has been used in China, but phase stability and vapor lock still need to be resolved in methanol-gasoline applications. In this paper, a series of tartaric esters were synthesized and used as phase stabilizers and saturation vapor pressure depressors for methanol-gasoline.

Results: The results showed that the phase stabilities of tartaric esters for methanol-gasoline depend on the length of the alkoxy group. Several tartaric esters were found to be effective in various gasoline-methanol blends, and the tartaric esters display high capacity to depress the saturation vapor pressure of methanol-gasoline.

Conclusion: According to the results, it can be concluded that the tartaric esters have great potential to be bifunctional gasoline-methanol additives.
\end{abstract}

Keywords: Methanol-gasoline, Tartaric esters, Phase stability, Evaporation

\section{Background}

Rapidly rising world demand, together with the limited oil supply, means developing clean and alternative fuels increasingly draws worldwide attention [1]. Among the alternative fuels, methanol displays fine combustion properties similar to gasoline and has such advantages as high octane number, low emissions, antiknock, rich resource, and mature technology, so it can be used as an alternative fuel for gasoline [2]. In recent years, extensive research of the low percentage methanol-gasoline has been carried out, and it has been applied in Shanxi, Sichuan, Zhejiang, Inner Mongolia, Shaanxi, Xinjiang and other provinces of China gradually [3]. However, there are several problems needing to resolve in methanol-gasoline application, in which the phase stability is the first and most important one. One of the popular solutions is to add phase stabilizer to reduce alcohol-oil interfacial tension $[4,5]$, such as ethers, ketones, esters, fatty alcohols, aliphatic hydrocarbons, fatty acids, non-ionic surfactants, acetal/ketones, biodiesels

\footnotetext{
*Correspondence: tangying78@xsyu.edu.cn

${ }^{1}$ College of Chemistry and Chemical Engineering, Xi'an Shiyou University, Xi'an, Shaanxi 710065, People's Republic of China

Full list of author information is available at the end of the article
}

and amidines [6-8]. Secondly, the low boil point of methanol leads to high possibility of vapor lock by raising the vapor pressure of methanol-gasoline [9-11]. The current solution for vapor lock is to add pressure depressor, such as aliphatic ketones, lynn classes, fatty aldehydes, fatty ethers, acetals/ketals, etc. At present, few researches have researched bifunctional additives with the functions of improving the phase stability as well as depressing the effective vapor pressure of the methanol-gasoline mixtures. In this work, a series of tartaric esters was synthesized and screened in the methanol-gasoline as a bifunctional additive for the phase stability and vapor depressor.

\section{Finding}

Tartaric esters were synthesized by two methods with high yield. The purified tartaric esters were evaluated as phase stabilizer and saturation vapor pressure depressor of methanol-gasoline. The results show that the efficiency depends on the length of the tartaric esters' alkoxy group. With the dosage of $0.1 \%$, all tartaric esters can depress the saturation vapor pressure lower than that of gasoline, and decyl tartaric is the most effective one.
(C) Chemistry Central

(c) 2014 Zhang et al.; licensee Chemistry Central Ltd. This is an Open Access article distributed under the terms of the Creative Commons Attribution License (http://creativecommons.org/licenses/by/2.0), which permits unrestricted use, distribution, and reproduction in any medium, provided the original work is properly credited. 


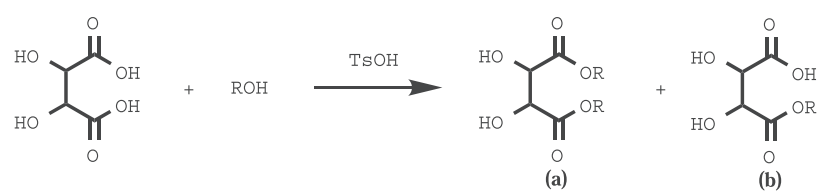

Scheme 1 The reaction of tartaric acid and alcohol.

\section{Experimental}

\section{Materials and methods}

All solvents were AR grade and purchased from Xi'an Chemical Agent $\mathrm{Co}$, and the $93^{\#}$ gasoline is commercially available. The phase stabilizing and pressure reducing tests were carried out on DFY-cryostat instrument (Xi'an Yuhui Instrument Co.Ltd.) and DSL-080 vapor pressure detector (Dalian the Ceon Electronic Equipment Co.Ltd.).

\section{Synthesis of tartaric esters}

The short-chain alcohols can co-dissolved with water, so the produced water can not be separated from the reactant, while long-chain alcohol can carry the produced water out under reflux. So different methods were used for the synthesis of tartaric esters.

Method A [12]: Tartaric acid (23 g), methanol $(18 \mathrm{~mL})$, and $p$-toluenesulfonic acid (TsOH) (0.6 g) were added in a $250 \mathrm{ml}$ flask. Refluxing for $10 \mathrm{~h}$, the mixture was cooled to room temperature. Methanol and methyl tartaric were distillated respectively. The synthesis of ethyl tartaric and propyl tartaric is similar to method above.

Method B [13,14]: Tartaric acid (0.15 mol), n-butanol (0.45 mol), cyclohexane $(30 \mathrm{~mL}), \quad p$-toluenesulfonic acid $(0.5 \mathrm{~g})$ were added in a $250 \mathrm{ml}$ flask equipped with a water separator. Refluxing for $5 \mathrm{~h}$, the mixture was cooled to room temperature, cyclohexane, $n$-butanol and $n$-butyl tartaric were separated by vacuum distillation. The synthesis of amyl tartaric,

Table 1 The results of the synthesis of tartaric esters

\begin{tabular}{cccc}
\hline Esters & Tartaric acid : alcohol & Method & Yield (\%) \\
\hline Methyl tartaric & $1: 30$ & A & 70.9 \\
Ethyl tartaric & $1: 30$ & A & 71.1 \\
Propyl tartaric & $1: 30$ & A & 76.5 \\
Butyl tartaric & $1: 5$ & B & 77.6 \\
Amyl tartaric & $1: 5$ & B & 64.5 \\
Hexyl tartaric & $1: 5$ & B & 60.2 \\
Hepyl tartaric & $1: 5$ & B & 72.1 \\
Octyl tartaric & $1: 5$ & B & 71.5 \\
Decyl tartaric & $1: 5$ & B & 72.2 \\
\hline
\end{tabular}

hexyl tartaric, hepyl tartaric, octyl tartaric, decyl tartaric is similar to method above.

\section{Phase stability test}

The fuel blends were prepared by blending 15, 30, 50 and 65 vol. \% of methanol with base gasoline, and the gasoline blends were assigned as M15, M30, M50 and M65. The phase stabilizing tests were carried out according to Chinese National standards of GB 801787, GB/T 23799-2009, DB61/T 352-2004 and DB51/ $\mathrm{T}$ 448-2004. First the test tube full of methanolgasoline with different ratios was placed in a cryostat, and then the temperature was adjusted from $40^{\circ} \mathrm{C}$ to $-25^{\circ} \mathrm{C}$. At each degree, the tube was taken out and was shaken for two to three seconds, and the phase separation temperature was determined as the solution becomes cloudy $[15,16]$. The tests were repeated until the separation temperature does not change for 3 times.

\section{Vapor pressure test}

Effect of tartaric esters on vapor pressure of methanolgasoline was investigated according to Chinese standards of GB 8017-87. The methanol-gasoline was poured into the vapor pressure detector and put into the water bath of $37.8^{\circ} \mathrm{C}$. The methanol-gasoline was intensive mixed by taking the detector from the water bath every $5 \mathrm{~min}$ and reversing violently. The operation

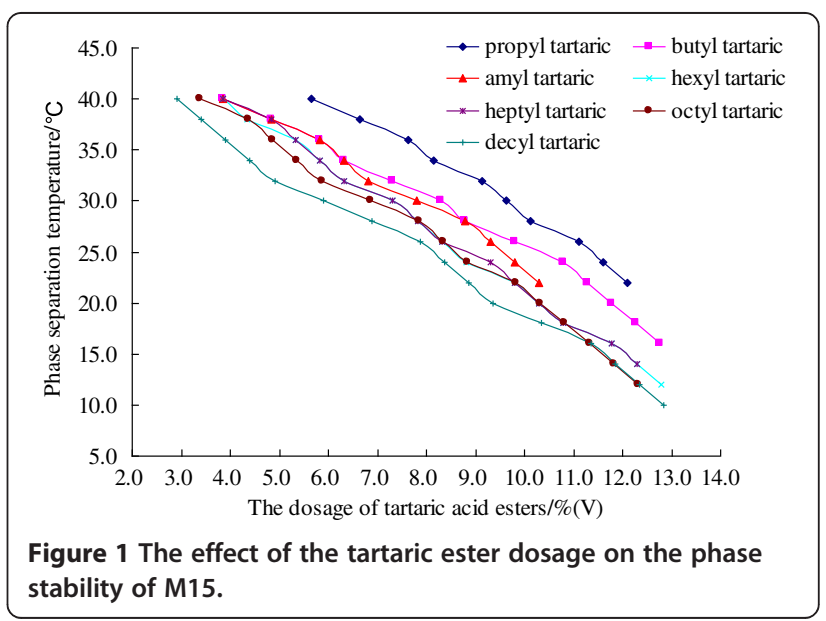




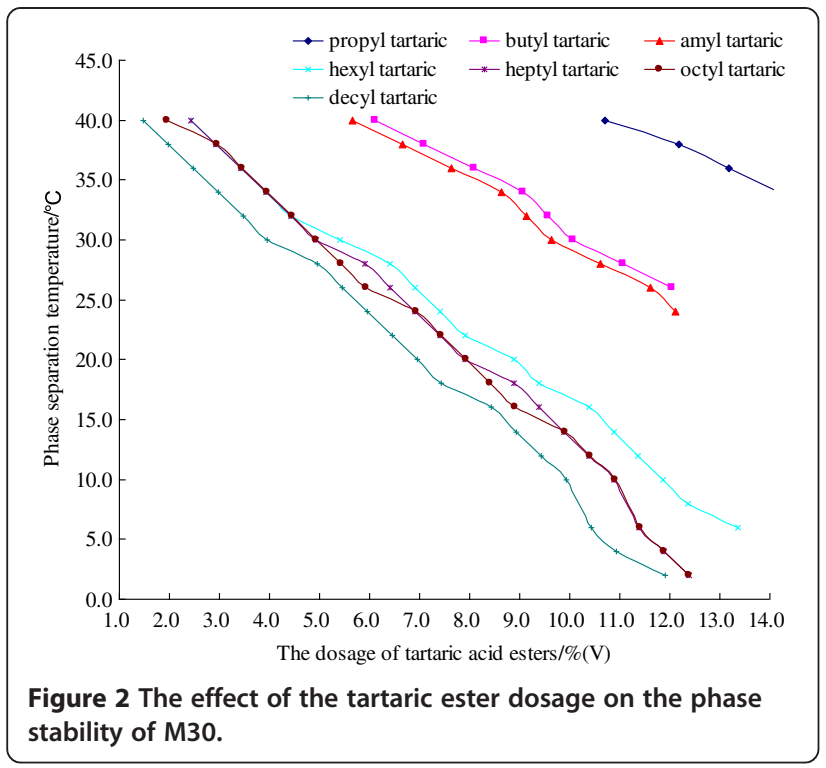

was repeated until the pressure does not change for 3 times.

\section{Results and discussion}

\section{Synthesis of tartaric esters}

The reactions of tartaric acid and alcohols are shown in Scheme 1, and both the reaction conditions and the yield are summarized in Table 1 . In this reaction, diester (a) is the main product, and the monoester (b) is the undesired byproduct. To reduce the byproduct, high quantities of alcohol are used. For the synthesis of the first three esters, the alcohols were employed with high ratio over 30: 1 to tartaric acid. For the rest esters, less alcohol is needed with the 5: 1 molar ratio of alcohol to tartaric acid, because the alcohols with more than 4 carbon atoms can compose azeotrope with water which can be separated by a water separator, so less alcohol is needed. To get purified tartaric esters for the following experiments phase stability and vapor pressure test, the synthesized esters were purified by vacuum distillation. The yields were obtained in the range from $60.2 \%$ to $77.6 \%$ as shown in Table 1 [17-19].

\section{Effect of tartaric ester on the phase stability of methanol- gasoline}

The phase stabilities of tartaric esters for the methanol-gasoline blends of M15, M30, M50 and $\mathrm{M} 65$ at different temperatures from $-25^{\circ} \mathrm{C}$ to $40^{\circ} \mathrm{C}$ were investigated and summarized in Figures 1, 2, 3 and 4. The results indicate that the length of alkoxy group of tartaric ester effects on the phase stability for methanol-gasoline significantly. For the esters with short alkoxy group, such as methyl tartaric and ethyl tartaric, the phase stability for methanol-gasoline are ineffective, even as the dosage over $10 \%$, methanol and gasoline does not homogenize to produce M15, M30, M50 and M65 at $40^{\circ} \mathrm{C}$. The reason may be due to the strong hydrophilicity but weak lipophilicity of short-carbon-chained tartaric ester, leading them hard to dissolve in gasoline. By increasing the carbon chain of tartaric esters, lipophilicity of the esters is markedly enhanced, and the dissolvent in gasoline is intensified, resulting in higher solubility for the various blends. According to the results, it can be found that long-carbon-chained tartaric esters are effective in phase stability to methanol-gasoline. The phase separation temperatures of the four methanol-gasoline blends with the ester dosage of $10 \%$ were estimated and shown in Figure 5. It can be found that the phase separation temperature declines along with the length of the alkoxy group. For M15, the lowest phase separation temperature was obtained as hexyl tartaric ester was employed. For M30, the optimal

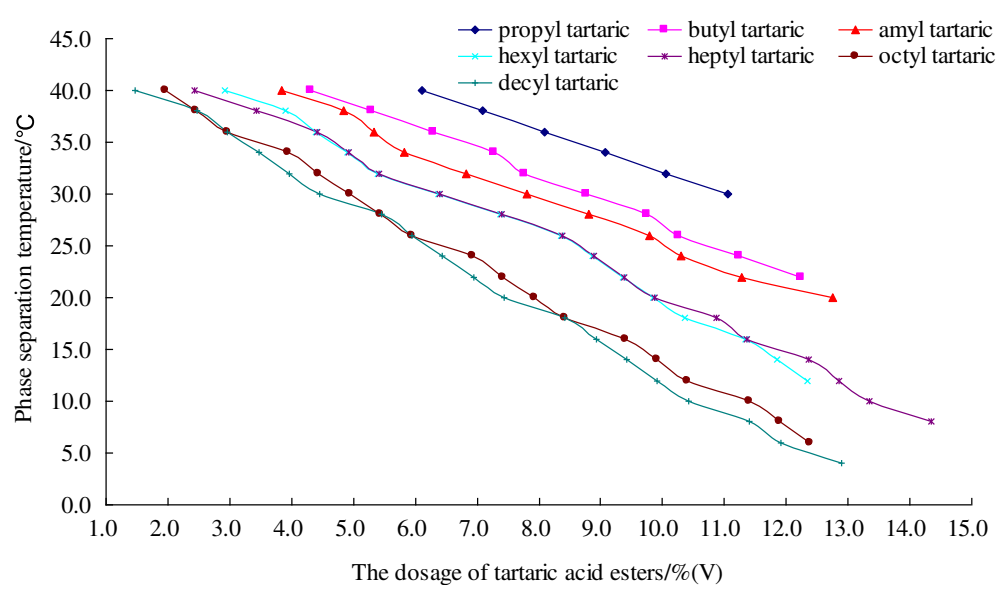

Figure 3 The effect of the tartaric ester dosage on the phase stability of M50. 


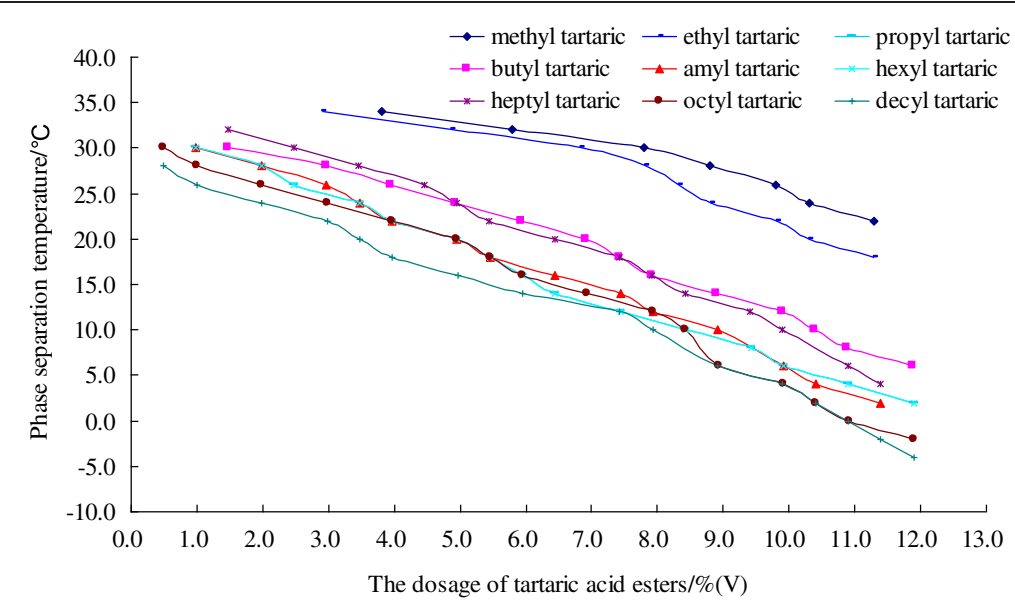

Figure 4 The effect of the tartaric ester dosage on the phase stability of M65.

carbon atom number of alkoxy group is 7. For M50, the optimal carbon atom number of alkoxy group is 8. For M65, the lowest phase separation temperature was obtained as the carbon atom number of alkoxy group is 8 .

\section{The effect of tartaric ester on the evaporation of methanol-gasoline}

The saturation vapor pressure will rise extremely over gasoline as it blends with low percentage methanol such as M15 and M30, which will lead to vapor block as it used under relative high temperature. Some chemicals with lower saturation vapor pressure have been employed to depress the high pressure of gasoline. In this work, the effect of tartaric ester on the saturation vapor pressure of M15 methanol-gasoline was investigated referred to GB 8017-87 "petroleum products the

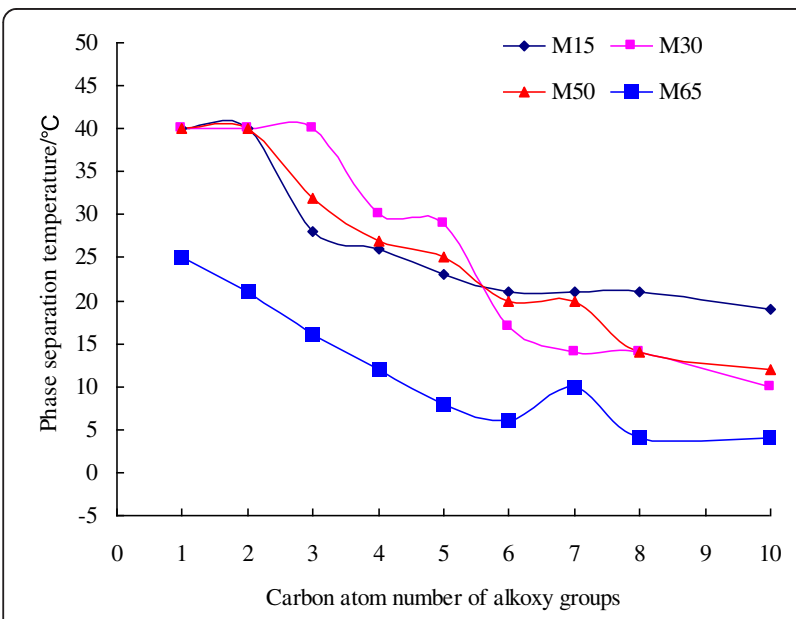

Figure $\mathbf{5}$ The relationship of the alkoxy groups and the phase separation temperature. vapor pressure determination method (Reid Method)", and the results are shown in Figure 6. The original saturation vapor pressure of M15 is $63.5 \mathrm{kPa}$, which is 5.7 $\mathrm{kPa}$ higher than that of gasoline. As little amount of esters were added in, the saturation vapor pressure was depressed obviously. With the esters' dosage of $0.1 \%$, methyl tartaric, ethyl tartaric, propyl tartaric, butyl tartaric, amyl tartaric, hexyl tartaric and decyl tartaric can depress the saturation vapor pressure lower than that of gasoline, among which decyl tartaric is the most effective one. Further increase of the dosage depresses the saturation vapor pressure ineffectively. The main reason may contribute to the distribution of tartaric esters on the surface of methanol-gasoline as shown in Figure 7. The tartaric esters alkoxy groups point to the hydrophobic part, gas phase, and the hydroxyl groups and the carboxyl groups point to the less hydrophobic part, methanol-gasoline, linking each other by hydroxyl

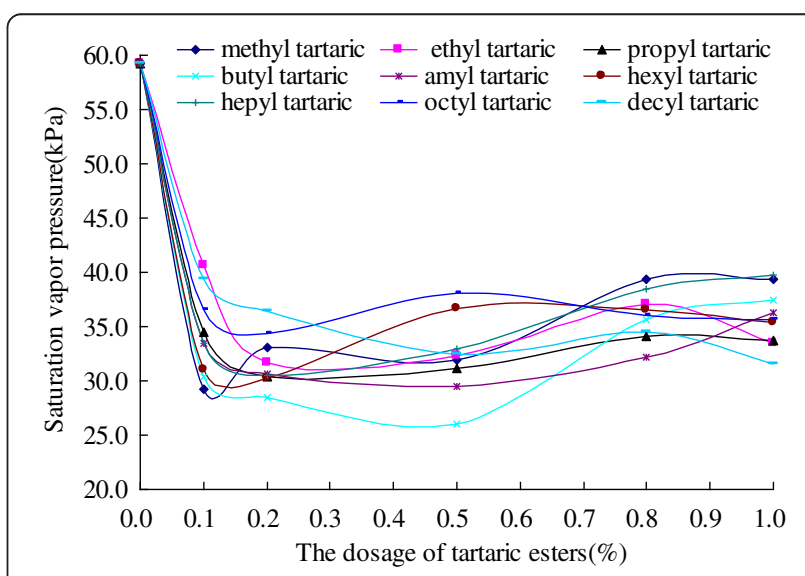

Figure 6 The effect of tartaric esters on the evaporation of methanol-gasoline M15 system. 


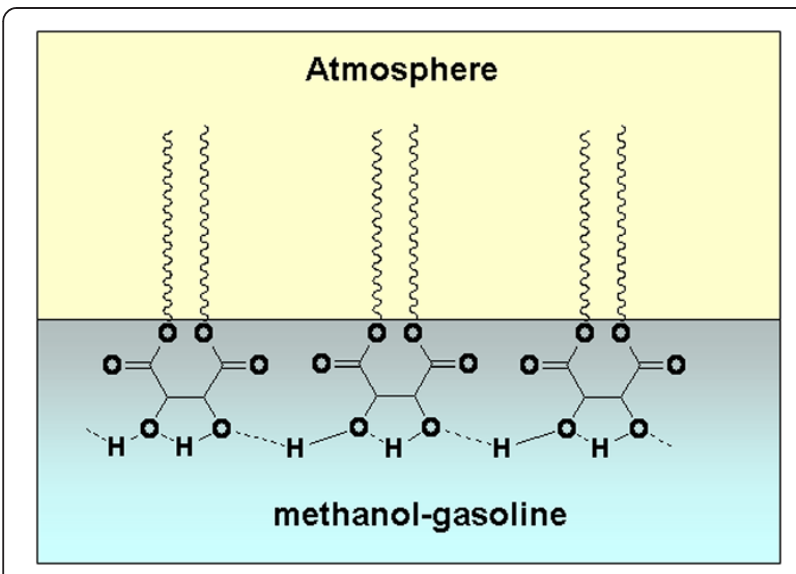

Figure 7 Distribution of ester molecules on the interface of methanol-gasoline and atmosphere.

bounds, which can prevent the formation of an azeotrope with low boiling point.

\section{Conclusions}

Tartaric esters were synthesized and screened for their performances of phase stabilizing in M15, M30, M50 and M65 and pressure reducing in M15. The results show that the length of alkoxy group of tartaric esters effects on the phase stability of methanol-gasoline significantly. The phase stability of tartaric esters for the methanol-gasoline system with long length is more potent than that with short length. All of the synthesized esters are potent to depress the saturation vapor pressure of methanol-gasoline. With the dosage of $0.1 \%$, all tartaric esters can depress the saturation vapor pressure lower than that of gasoline, and decyl tartaric is the most effective one.

\section{Competing interests}

The authors declare that they have no competing interests.

\section{Authors' contributions}

Jie Zhang carried out the synthesis of tartaric esters, and Changchun Yang participated in using phase stabilizer and saturation vapor pressure depressor of methanol-gasoline. Ying Tang, Rui Zhou, Lianghong Xu and Xiaoli Wang evaluated that the stabilities of the methanol-gasoline depend on the length of the tartaric esters'alkoxy group. All authors have read and approved the final manuscript.

\section{Acknowledgements}

Financial support from National Science Foundation of China (21306149), Scientific Research Program Funded by Shaanxi Provincial Education Department (2013JK0646).

\section{Author details}

${ }^{1}$ College of Chemistry and Chemical Engineering, Xi'an Shiyou University, Xi'an, Shaanxi 710065, People's Republic of China. ${ }^{2}$ College of Petroleum Engineering, China University of Petroleum-Beijing, Beijing 102249, People's Republic of China. ${ }^{3}$ Shaanxi Yanchang Zhongli New Energy Co. Ltd, Xi'an, Shaanxi 710065, People's Republic of China.

\section{References}

1. Liang W: Development of the research and application of methanol gasoline. Chin Foreign Energy 2006, 11(2):95-96.

2. He XL, Zhan YH, Li SS: Fuel of Internal Combustion Engine. Beijing: China Petrochemical Press; 1999:352-392.

3. Yang JJ, Huang HB: Low percentage of methanol gasoline evaporation. Intern Combust Engine Power Equip 2007, 4:40-41.

4. Fu WH: Research on miscibility of methanol-gasoline. Learned Journal of Petroleum 1986, 2(3):69-78.

5. He P: Research and Application of the Additive of Methanol Gasoline. Dalian: Liaoning Normal University; 2010:9-10.

6. Qiao YB: Discussion on the problem of methanol gasoline. Contemp Petrol Petrochemical 2003, 11(06):7-8.

7. Cao GZ, Tan SY: Research on Application of Methanol Gasoline. Chongqing: Chongqing University; 2008:1-2.

8. Wang YZ: Physical and Chemical Properties of the Vehicle of Methanol Gasoline. Xi'an: Chang'an University; 2006:28-31.

9. Wang MQ, Hu YB: Study on evaporation of low percentage of methanol gasoline. Guang Zhou Chem 2010, 39(2):87-89.

10. Luo $T$, Yang L: Study on the basis of gasoline for the impact of vapor lock of the methanol gasoline. Times J Chem 2005, 19(4):1-2.

11. Guo HJ: Development of gasoline detergent additive research. Polym Mater Sci Eng 2002, 18(1):171-175.

12. Hu AX, Zhao HT, Fan GZ: The ion exchange resin catalyzed and synthesized of (2R,3R)-tartaric acid dimethyl. Learned J Hunan Univ 1998, 25(1):23-26.

13. Wu D, Wang GS: Study on L-(+)-tartaric acid diethyl. Chem Eng 2009, 37(9):1-3.

14. Wei Y, Han XQ, Li J: Synthesis of L-tartaric acid $n$-butyl. Chem Eng 2007, $4: 1-2$.

15. Zhang J, Yang CC, Tang Y: Advances in stability and detergence of methanol gasoline blends. Speciality Petrochemicals 2011, 28(6):66-70.

16. Xi Q, Li CG, Xu FL: Study on the phase stability methanol-gasoline system. Times J Chem 2007, 21(8):10-12.

17. Chen GB, Tang KW, Chen TH: Synthesis of L-tartaric acid hexyl ester. Synthetic Chem 2004, 12:207-209.

18. Chen GB, Tang KW, Wang CG: Synthesis of D-tartaric acid heptane esters. Synthetic Chem 2006, 14(1):82-83.

19. Tang KW, Chen GB, Li JL: Synthesis of D-tartaric acid octyl esters. Synthetic Chem 2005, 13(1):77-79.

doi:10.1186/1752-153X-8-25

Cite this article as: Zhang et al:: Development of tartaric esters as bifunctional additives of methanol-gasoline. Chemistry Central Journal $20148: 25$.

\section{Publish with ChemistryCentral and every scientist can read your work free of charge \\ "Open access provides opportunities to our colleagues in other parts of the globe, by allowing anyone to view the content free of charge." W. Jeffery Hurst, The Hershey Company. \\ - available free of charge to the entire scientific community \\ - peer reviewed and published immediately upon acceptance \\ - cited in PubMed and archived on PubMed Central \\ - yours - you keep the copyright \\ Submit your manuscript here: \\ http://www.chemistrycentral.com/manuscript/ \\ (1) \\ Chemistry Central}

